\title{
Retrospective Case Series of Patients with Diabetes or Prediabetes Who Were Switched from Omega-3-Acid Ethyl Esters to Icosapent Ethyl
}

\author{
Amir Hassan • Nadeem Tajuddin • Ali Shaikh
}

To view enhanced content go to www.cardiologytherapy-open.com Received: November 18, 2014 / Published online: December 17, 2014

(C) The Author(s) 2014. This article is published with open access at Springerlink.com

\begin{abstract}
Introduction: Patients with diabetes and prediabetes are at increased risk of dyslipidemia and cardiovascular disease. To reduce this risk, statins and additional therapies may be considered. Omega-3 fatty acids offer an option to reduce triglycerides (TG) and potentially improve other lipid parameters, although products that contain docosahexaenoic acid (DHA) may increase low-density lipoprotein cholesterol (LDL-C) while eicosapentaenoic acid (EPA) does not. Prescription formulations include omega-3-acid mixtures (combination of predominantly EPA and DHA), and icosapent ethyl (high-purity prescription form of EPA ethyl ester); prescription omega-3 products are indicated as an adjunct to diet to reduce TGs in adult patients with severe hypertriglyceridemia at a dose of $4 \mathrm{~g}$ /day.
\end{abstract}

A. Hassan $(\bowtie) \cdot N$. Tajuddin · A. Shaikh

SE Memorial Clinic, 11914 Astoria Blvd, Suite 330,

Houston, TX 77089, USA

e-mail: amirhassanmd@gmail.com
Methods: This was a retrospective analysis of records from a private endocrinology practice of patients who received omega-3-acid ethyl esters (OM3EE) (4 g/day) and were subsequently switched to icosapent ethyl (IPE; $4 \mathrm{~g} /$ day) due to the potential of OM3EE to raise LDL-C and/or cause gastrointestinal upset. Patient records were analyzed for LDL-C, TG, total cholesterol (TC), high-density lipoprotein cholesterol (HDL-C), and non-HDL-C measured before and after the switch to IPE.

Results: The records of ten patients met the criteria for this analysis and were included. All patients had taken OM3EE for $\geq 1$ year prior to their last lipid measurement before switching to IPE, and all had been taking IPE for $>3$ months at the time of their subsequent lipid measurement. Nine of the ten patients were on concomitant statin therapy throughout. Reductions in LDL-C, TC, and non-HDL-C were observed in eight patients, reductions or no changes in TG were observed in eight patients, and increases or no changes in HDL-C were observed in eight patients. No gastrointestinal adverse events were observed.

Conclusion: In most patients with prediabetes or diabetes who switched from OM3EE to IPE, 
LDL-C and other lipid parameters improved. IPE was well tolerated.

Keywords: Diabetes; Docosahexaenoic acid; Dyslipidemia; Eicosapentaenoic acid; Icosapent ethyl; Lovaza; Low-density lipoprotein cholesterol; Triglycerides; Vascepa

\section{INTRODUCTION}

Dyslipidemia is common in patients with diabetes and prediabetes and is a risk factor for cardiovascular disease (CVD) [1]. Medical guidelines from the American Diabetes Association (ADA) and the American Association of Clinical Endocrinologists (AACE) recommend statins for the pharmacologic management of dyslipidemia in patients with diabetes or prediabetes $[1,2]$.

Despite the beneficial effects of statins on low-density lipoprotein cholesterol (LDL-C), some patients may have a residual risk of cardiovascular (CV) events, CV death, and myocardial infarction due to the effects of elevated triglycerides (TGs) [3, 4], as statins achieve only a $10-30 \%$ reduction in TGs [5]. The Residual Risk Reduction Initiative defines residual $\mathrm{CV}$ risk as the risk of $\mathrm{CV}$ events that persists despite achievement of LDL-C, blood pressure, and glycemic treatment goals [6]. Thus, add-on therapy may be needed to control TGs and TG-rich lipoproteins and to further reduce risk in some statin-treated patients. Such adjunct therapies include omega-3 fatty acids (OM3FAs) [2].

The OM3FAs eicosapentaenoic acid (EPA) and docosahexaenoic acid (DHA) have numerous known CV benefits in patients with dyslipidemia such as antidysrhythmic, antiatherogenic, antiinflammatory, antithrombotic, and antihypertensive effects, and in particular, reduction of TGs $[7,8]$. OM3FA therapies are approved by the United States Food and Drug Administration for use as adjunct to diet to reduce TGs in adult patients with severe ( $\geq 500 \mathrm{mg} / \mathrm{dL}$ ) hypertriglyceridemia. Approved formulations commercially available at the time of this analysis were omega-3-acid ethyl esters (OM3EE; Lovaza ${ }^{\circledR}$; GlaxoSmithKline, Research Triangle Park, North Carolina, USA), a formulation that contains a combination of the ethyl esters of EPA and DHA [9], and icosapent ethyl (IPE; Vascepa ${ }^{\circledR}$; Amarin Pharma Inc., Bedminster, New Jersey, USA), a high-purity prescription form of EPA ethyl ester [10]. Each is administered as a daily dose of $4 \mathrm{~g}$. Notably, OM3FA formulations that contain DHA have been associated with increases in LDL-C, but EPA does not increase LDL-C [11]. Similarly, the prescribing information of OM3EE warns that increases in LDL-C have occurred in some patients and recommends periodic LDL-C monitoring [9], while the prescribing information of IPE does not contain this warning.

Based on the known beneficial effects of OM3FAs, and in an effort to reduce TG and/or $\mathrm{CV}$ risk in patients in our private endocrinology practice, we began prescribing OM3EE to patients based on our overall assessments of their clinical status and potential for CV risk. In addition to the known possible increase in LDL$\mathrm{C}$ associated with OM3EE use and consistent with the Adverse Reactions section of the OM3EE prescribing information [9], we noted that gastrointestinal problems and fishy eructation [12] were among the side effects that interfered with compliance in our practice. When IPE became commercially available in 2013, we noted that it contained purified EPA, which could reduce TG without the increases in LDL-C associated with OM3EE, and that the incidence of eructation and gastrointestinal 
upset in statin-treated patients was lower than that seen in statin-treated patients receiving placebo [10, 13, 14]. In our clinical judgment, IPE had the potential for fewer side effects and to be better tolerated, with no adverse effects on LDL-C, so we began switching our patients from OM3EE to IPE. The objective of the current analysis was to retrospectively assess the lipid profiles of adult patients with diabetes or prediabetes who had been receiving OM3EE and were subsequently switched to IPE.

\section{METHODS}

\section{Study Design}

This was a retrospective chart review of patients in a private endocrinology practice in Houston, Texas. Records for diabetic and prediabetic patients who had been receiving OM3EE and subsequently underwent a switch to IPE were identified through a search of Electronic Clinical Works (ECW)/Electronic Health Records (EHR). The authors received approval from the Western Institutional Review Board, Puyallup, WA, for the conduct of this study. This article does not contain any new studies with human or animal subjects performed by any of the authors.

\section{Patients and Treatment}

Patients were eligible if they had well-controlled diabetes or prediabetes and hyperlipidemia; had been switched from OM3EE $4 \mathrm{~g}$ /day to IPE $4 \mathrm{~g}$ / day; had been taking IPE for more than 2 months; had available lipid measurements; and had been clinically stable over the course of the period examined. Patients were excluded if they had poor thyroid function, uncontrolled diabetes, insulin use, gaps in treatment, missing laboratory values, known noncompliance, changes in lipid-lowering medications, or were taking niacin, fibrates, bile acid sequestrants, or other OM3FA products (including dietary supplements). Use of ezetimibe was permitted.

\section{Assessments}

Fasting blood samples were collected from patients and analyzed at local branches of large, national clinical laboratories (either Quest Diagnostics or LabCorp) according to patient insurance coverage. Lipid measurements [LDL-C, TG, total cholesterol (TC), high-density lipoprotein cholesterol (HDL-C), and non-HDL-C] were analyzed at dates prior to initiation of OM3EE, at the latest date with available data prior to switching from OM3EE to IPE, and at least 2 months after the switch from OM3EE to IPE. Percent changes were calculated from measurements at latest date available while on OM3EE to measurements made while on IPE. LDL-C assessments were calculated by the clinical laboratory. Non-HDL-C was calculated as TC minus HDL-C [15]. Lipid values for individual patients while on OM3EE and after the switch to IPE were plotted for visualization of effects.

\section{RESULTS}

\section{Patients}

Of the patient records retrieved from the ECW/ EHR database, ten met the inclusion criteria and were analyzed. The population included eight adult males and two adult females ranging in age from 42 to 66 years (Table 1) who initiated OM3EE treatment between June 2007 and August 2012 and switched to IPE treatment between January and June 2013. Nine of the ten 


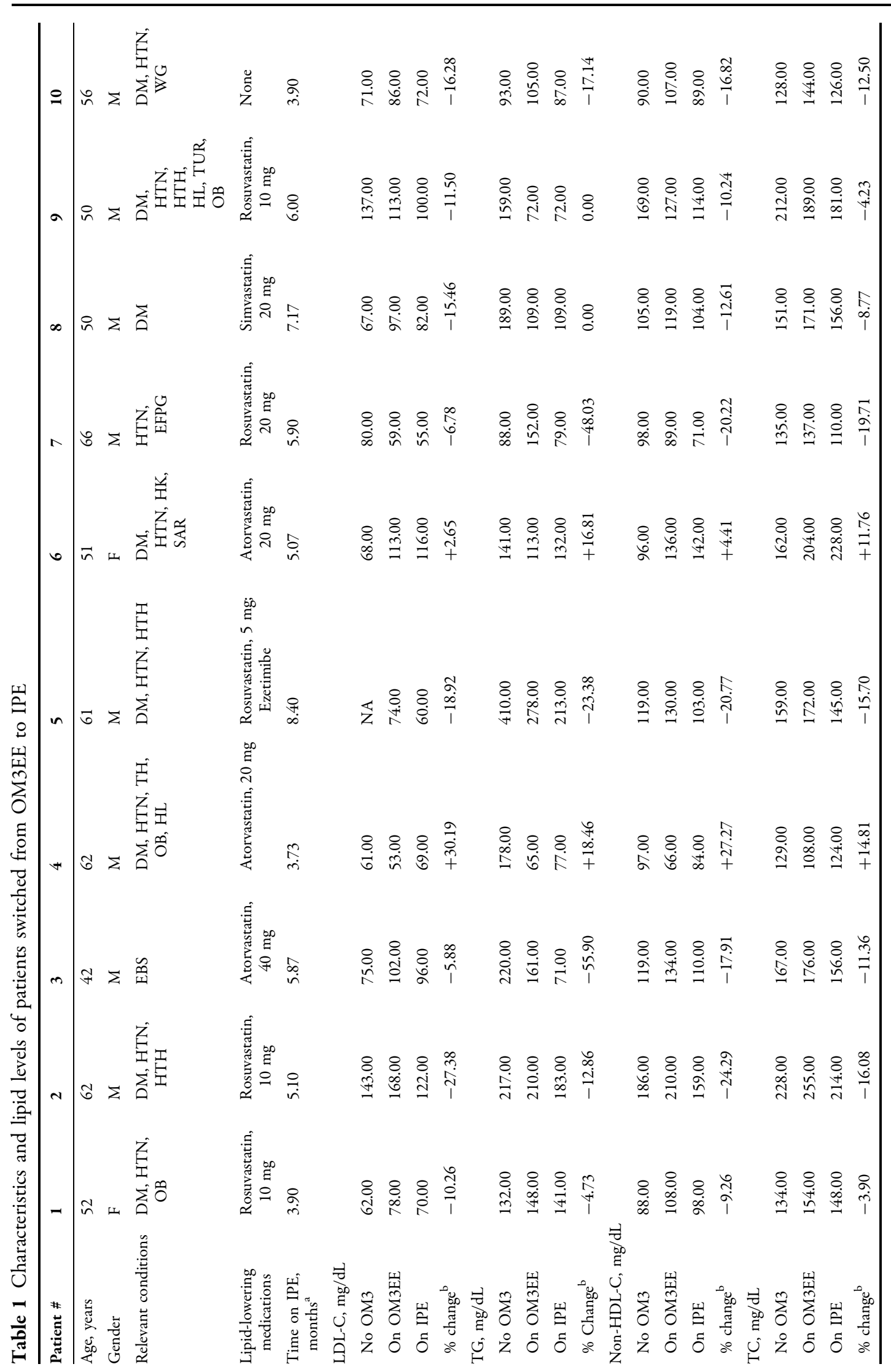




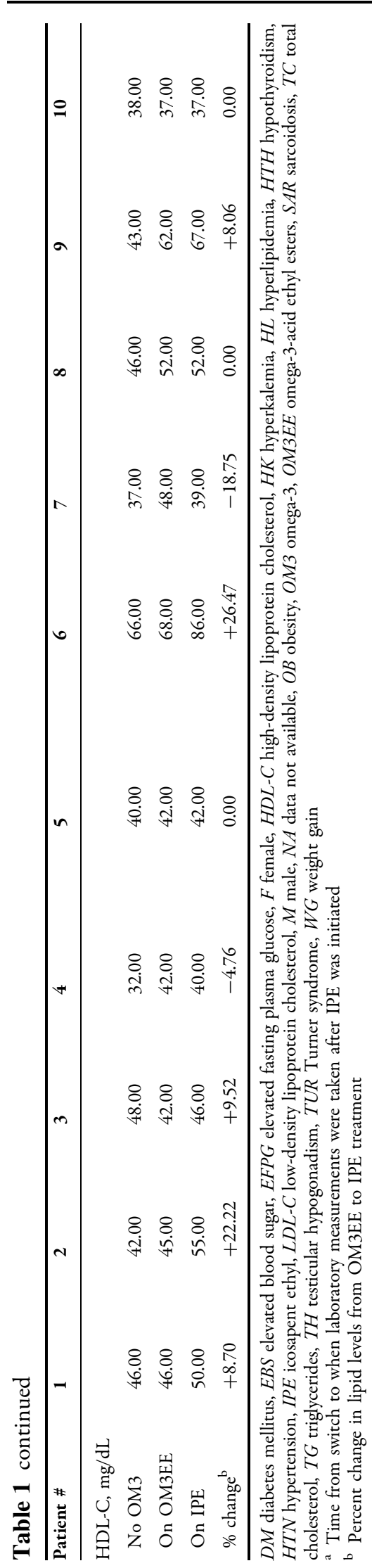

patients were receiving a statin (rosuvastatin, atorvastatin, or simvastatin); one statin-treated patient was also receiving ezetimibe (Table 1 ). Other medications are listed in Table 2; no changes in medications that would affect cholesterol or TGs occurred during the course of treatment with OM3EE or IPE. All patients had been taking OM3EE for $\geq 1$ year (range 1.0-5.6 years) prior to measurement of lipid levels while on OM3EE and all had been taking IPE for $>3$ months (range 3.9-8.4 months) prior to measurement of lipid levels while on IPE. The time elapsed between the measurements taken while on OM3EE and while on IPE ranged from 4.0 to 25.7 months.

Diabetes had been diagnosed in eight patients, one of the remaining patients had elevated fasting plasma glucose, and the other was noted as having elevated blood sugar (Table 1). Eight patients also had hypertension (Table 1).

Following the switch from OM3EE to IPE, no gastrointestinal adverse events or fishy odor/ eructation were reported. IPE was well tolerated.

\section{Effects on Lipid Parameters}

Percentage changes in lipid values after the switch from OM3EE to IPE are shown in Table 1. Lipid levels for patients while on OM3EE before the switch to IPE ranged from 53 to $168 \mathrm{mg} / \mathrm{dL}$ for LDL-C, 65 to $278 \mathrm{mg} / \mathrm{dL}$ for TG, 66 to $210 \mathrm{mg} / \mathrm{dL}$ for non-HDL-C, 108 to $255 \mathrm{mg} / \mathrm{dL}$ for $\mathrm{TC}$, and 37 to $68 \mathrm{mg} / \mathrm{dL}$ for HDL-C. Lipid levels for patients while on IPE ranged from 55 to $122 \mathrm{mg} / \mathrm{dL}$ for LDL-C, 71 to $213 \mathrm{mg} / \mathrm{dL}$ for $\mathrm{TG}, 71$ to $159 \mathrm{mg} / \mathrm{dL}$ for non-HDL-C, 110 to $228 \mathrm{mg} / \mathrm{dL}$ for TC, and 37 to $86 \mathrm{mg} / \mathrm{dL}$ for HDL-C.

Figure 1 summarizes the individual lipid levels before and after the switch from OM3EE to IPE. Reductions in LDL-C, TC, and non-HDL-C 
Table 2 Other medications

Antidiabetics

Antihypertensives

Dietary supplements

Others
Exenatide, glimepiride, glipizide, liraglutide, metformin, pioglitazone, saxagliptin, sitagliptin

Bisoprolol, clonidine, irbesartan, lisinopril, losartan, metoprolol, olmesartan

Folic acid, glucosamine, vitamin B12, vitamin D (with or without calcium)

Adefovir, aspirin, celecoxib, duloxetine, levothyroxine, loratadine, testosterone gel, vardenafil were observed in eight patients, reductions or no changes in TG were observed in eight patients, and increases or no changes in HDL-C were observed in eight patients.

\section{DISCUSSION}

In this retrospective analysis of patients with diabetes or prediabetes, we sought to evaluate the lipid profiles in patients switched from OM3EE to IPE in our private clinical endocrinology practice. We found that, in most cases, patients experienced decreases in LDL-C, TG, non-HDL-C, and TC and increases in HDL-C following the switch to IPE. Two patients (numbers 4 and 6) experienced increases in LDL-C, TG, non-HDL-C, and TC after switching to IPE, although lipid levels in patient 4 were still within the acceptable range as specified by the AACE [2] and the recent National Lipid Association (NLA) recommendations for patient-centered management of dyslipidemia [16]. In patient 6 , the increase in LDL-C was relatively small (from 113 to $116 \mathrm{mg} / \mathrm{dL}$ ), and TGs were still within the acceptable range as specified by the AACE and NLA. Overall, the effects seen represented improvements in the assessed lipid parameters in most patients.

Our findings are similar to those of other recent reports of patients switched from $\mathrm{EPA}+\mathrm{DHA}$ formulations to IPE. Case studies of two patients with type 2 diabetes and dyslipidemia who were switched from either a dietary supplement containing EPA + DHA or OM3EE to IPE showed improvements in LDL-C, TG, non-HDL-C, and nominal effects on HDL-C [17]. In addition, in a retrospective case series of 14 statin-treated patients with hyperlipidemia from a private practice in 4 Western New York locations, switching from OM3EE to IPE achieved reductions in LDL-C, TGs, non-HDL-C, and TC in most cases [18]. There were three patients in the Western New York study that had diabetes, and all three experienced reductions in non-HDL-C and TC following the switch from OM3EE to IPE. Two of the three patients with diabetes experienced TG reductions; one patient experienced an increase in TG from 62 to $81 \mathrm{mg} / \mathrm{dL}$, which may not be considered clinically significant, as $81 \mathrm{mg} / \mathrm{dL}$ is considered to be within the acceptable range as specified by the guidelines of the NLA [16], AACE [2], and the Endocrine Society [19]. Two of these three patients also experienced reductions in LDL-C, while one patient had a reported LDL-C increase of $39.8 \%$. However, upon calculating the expected LDL-C level in this patient based upon the Friedewald formula (LDL-C $=\mathrm{TC}$ minus HDL-C minus $\mathrm{TG} / 5$; all values given in $\mathrm{mg} / \mathrm{dL}$ ) [20], the LDL-C level in this patient while on OM3EE would be expected to have been $173 \mathrm{mg} / \mathrm{dL}$ and not $123 \mathrm{mg} / \mathrm{dL}$ as reported, resulting in an actual $0.6 \%$ decrease in LDL-C following the switch from OM3EE to IPE. 

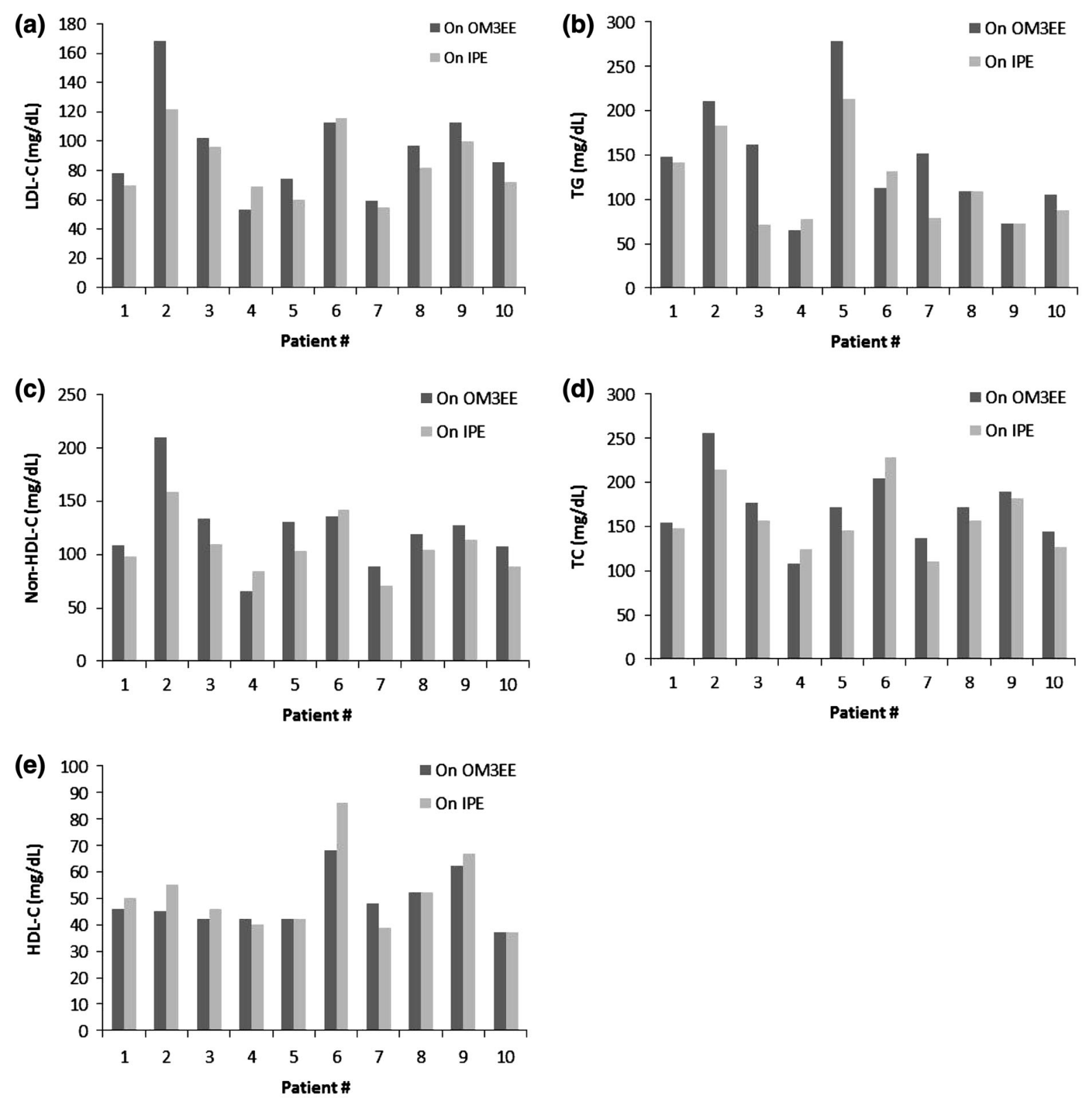

Fig. 1 Individual lipid parameters before and after the switch from omega-3-acid ethyl esters (OM3EE) to icosapent ethyl (IPE) in patients with diabetes/prediabetes. a Low-density lipoprotein cholesterol (LDL-C);

b triglycerides (TG); c non-high-density lipoprotein cholesterol (non-HDL-C); $\mathbf{d}$ total cholesterol (TC); and e high-density lipoprotein cholesterol (HDL-C)

The results of our retrospective analysis are consistent with the results of clinical trials of IPE for both efficacy and tolerability. The phase 3, multicenter, placebo-controlled, randomized, double-blind, 12-week ANCHOR (NCT01047501) study examined the safety and efficacy of IPE in

high-risk statin-treated patients with residually high TGs $(\geq 200$ and $<500 \mathrm{mg} / \mathrm{dL})$ despite statin control of LDL-C ( $\geq 40$ and $\leq 115 \mathrm{mg} / \mathrm{dL}$ ). In a subanalysis of patients with diabetes from the ANCHOR study, IPE 4 g/day significantly decreased median LDL-C by $6.3 \%(P=0.02)$, TGs 
by $23.2 \%(P<0.0001)$, non-HDL-C by $14.4 \%$ $(P<0.0001)$, TC by $12.7 \% \quad(P<0.0001)$, and HDL-C by $5.0 \% \quad(P<0.01)$ compared with placebo [21]. The decreases in LDL-C, TG, non-HDL-C, and TC in the present analysis are consistent with these results, with perhaps somewhat more robust reductions in LDL-C observed in our analysis. However, the increases in HDL-C in our analysis differ somewhat from the small but significant decreases in HDL-C in the ANCHOR subanalysis. It is unclear why this difference was observed, but may perhaps be attributable to differences in the patient populations, differences in concurrent medications, and/or other unknown factors. Similar to results of clinical trials where tolerability of IPE was comparable to placebo $[13,14]$, IPE was well tolerated in our patient population, with no gastrointestinal adverse events reported.

Our findings are novel in that we examined the effects of switching from OM3EE to IPE in patients with diabetes or prediabetes, an important patient population with respect to CVD risk. While patients in this analysis may not have been receiving a maximally approved statin dose, each patient was receiving their own maximally tolerated dose. Our experience has been that patients may have difficulty tolerating higher statin doses due to adverse effects such as muscle aches and fatigue. Diabetes in these patients was well controlled as were TGs in most cases.

It is our opinion that the unmet need of residual $\mathrm{CV}$ risk in our patients should be addressed, and thus we prescribe prescription OM3FA products in our endocrinology practice. The results of this analysis support switching such patients from OM3EE to IPE, including those receiving statin treatment. The TGlowering effects of OM3FAs are well established [22] and reducing very high TG is a well-accepted treatment approach to reduce the risk of pancreatitis [16]. However, results of OM3FA outcomes studies have been inconsistent or somewhat controversial [2332]. Disappointing results wherein lack of effect of OM3FAs on CV outcomes was observed may have been due in part to intervention with low doses of OM3FA ( $\sim 1-2$ g/day) in the context of background contemporary statin therapy $[26,27,32]$. Other factors may include differences in baseline CV risk, baseline TGs, and background dietary OM3FA intake. However, the results of the Japan EPA Lipid Intervention Study (JELIS) support long-term use of highly purified EPA with concomitant statin therapy for the primary and the secondary prevention of major coronary events $[28,29,33]$. In particular, a subgroup analysis of primary prevention in patients from JELIS demonstrated that the risk for major coronary events was particularly high in patients with high TGs and low HDL-C and that EPA potently suppressed major coronary events in these patients [29]. Furthermore, recent genetic studies of apolipoprotein C3 have suggested a causal role for TG-rich lipoproteins in the development of CVD [34, 35]. Thus, the role for OM3FAs in the prevention of $\mathrm{CV}$ events bears further investigation. The effects of IPE on $\mathrm{CV}$ outcomes are currently being evaluated in the ongoing Reduction of Cardiovascular Events with EPA-Intervention Trial (REDUCEIT; NCT01492361), a phase 3, randomized, parallel-assignment, double-blind safety and efficacy study [36]. In REDUCE-IT, patients are receiving IPE $4 \mathrm{~g} /$ day or placebo and have persistent hypertriglyceridemia despite statin treatment along with established CVD or high risk for CVD. The purpose of the trial is to 
investigate whether preventative therapy with IPE + statin is superior to statin therapy alone in the long-term reduction of $\mathrm{CV}$ events. The results of this trial are highly anticipated and should provide key insights into the role of TGlowering therapy in the reduction of $\mathrm{CV}$ risk and events. It will be the first trial of its kind to examine the effects of OM3FAs on CV outcomes in patients with persistently high TG.

The current analysis was exploratory and may be of interest to the clinical community for potential future prospective studies. The limitations of the current analysis are that it was conducted retrospectively with a small number of patients at a single endocrine clinic and that the same time points and clinical laboratory were not used for all lipid assessments. Other real-world limitations include lack of verification that lipids were measured in the fasted state and heterogeneity with regard to concomitant medications other than OM3EE and IPE and underlying medical conditions other than diabetes. Given the small sample size and patient heterogeneity, data were summarized descriptively. Further prospective and/or retrospective investigation would be helpful to better understand the effects of switching from OM3EE to IPE in patients with diabetes or prediabetes.

\section{CONCLUSIONS}

In this analysis of patients with prediabetes or diabetes in a private endocrinology clinical practice who were switched from OM3EE to IPE, LDL-C, TG, non-HDL-C, TC, and HDL-C improved in most patients. IPE was well tolerated after switching from OM3EE. Taken together, the evidence to date suggests that treatment with IPE produces beneficial effects in patients with diabetes who may also be receiving statin therapy, and that switching patients from OM3EE to IPE offers a therapeutic option that results in a beneficial lipid profile.

\section{ACKNOWLEDGMENTS}

Article processing charges for this study were funded by Amarin Pharma Inc., Bedminster, NJ, USA. Medical writing assistance was provided by Elizabeth Daro-Kaftan, PhD of Peloton Advantage, Parsippany, NJ, USA and funded by Amarin Pharma Inc. Medical review, scientific reference checks, and associated assistance were provided by Sumita Chowdhury, MD, MPH, and Sephy Philip, RPh, PharmD of Amarin Pharma Inc. All named authors meet the ICMJE criteria for authorship for this manuscript, take responsibility for the integrity of the work as a whole, and have given final approval to the version to be published.

Conflict of interest. Dr. Amir Hassan declares being a stock shareholder of Amarin Pharma Inc.

Drs. Ali Shaikh and Nadeem Tajuddin declare no conflicts of interest.

Compliance with ethics guidelines. The authors received approval from the Western Institutional Review Board, Puyallup, WA, for the conduct of this study. This article does not contain any new studies with human or animal subjects performed by any of the authors.

Open Access. This article is distributed under the terms of the Creative Commons Attribution Noncommercial License which permits any noncommercial use, distribution, and reproduction in any medium, provided the original author(s) and the source are credited. 


\section{REFERENCES}

1. American Diabetes Association. Standards of medical care in diabetes-2014. Diabetes Care. 2014;37 Suppl 1:S14-80.

2. Jellinger PS, Smith DA, Mehta AE, Ganda O, Handelsman Y, Rodbard HW, et al. American Association of Clinical Endocrinologists' guidelines for management of dyslipidemia and prevention of atherosclerosis. Endocr Pract. 2012;18(Suppl 1):1-78.

3. Murad MH, Hazem A, Coto-Yglesias F, Dzyubak S, Gupta S, Bancos I, et al. The association of hypertriglyceridemia with cardiovascular events and pancreatitis: a systematic review and metaanalysis. BMC Endocr Disord. 2012;12:2.

4. Miselli MA, Nora ED, Passaro A, Tomasi F, Zuliani G. Plasma triglycerides predict ten-years all-cause mortality in outpatients with type 2 diabetes mellitus: a longitudinal observational study. Cardiovasc Diabetol. 2014;13:135.

5. Miller M, Stone NJ, Ballantyne C, Bittner V, Criqui $\mathrm{MH}$, Ginsberg $\mathrm{HN}$, et al. Triglycerides and cardiovascular disease: a scientific statement from the American Heart Association. Circulation. 2011;123:2292-333.

6. Fruchart JC, Davignon J, Hermans MP, Al-Rubeaan $\mathrm{K}$, Amarenco P, Assmann G, et al. Residual macrovascular risk in 2013: what have we learned? Cardiovasc Diabetol. 2014;13:26.

7. Bays H. Fish oils in the treatment of dyslipidemia and cardiovascular disease. In: Kwiterovich PO, editor. The Johns Hopkins textbook of dyslipidemia. Philadelphia: Lippincott Williams \& Wolters Kluwer; 2010. p. 245-57.

8. Mozaffarian D, Wu JH. Omega-3 fatty acids and cardiovascular disease: effects on risk factors, molecular pathways, and clinical events. J Am Coll Cardiol. 2011;58:2047-67.

9. Lovaza [package insert]. Research Triangle Park: GlaxoSmithKline; 2014.

10. Vascepa [package insert]. Bedminster: Amarin Pharma Inc.; 2013.

11. Wei MY, Jacobson TA. Effects of eicosapentaenoic acid versus docosahexaenoic acid on serum lipids: a systematic review and meta-analysis. Curr Atheroscler Rep. 2011;13:474-83.

12. Harris WS, Ginsberg HN, Arunakul N, Shachter NS, Windsor SL, Adams M, et al. Safety and efficacy of
Omacor in severe hypertriglyceridemia. J Cardiovasc Risk. 1997;4:385-91.

13. Bays HE, Ballantyne CM, Kastelein JJ, Isaacsohn JL, Braeckman RA, Soni PN. Eicosapentaenoic acid ethyl ester (AMR101) therapy in patients with very high triglyceride levels [from the Multicenter, plAcebo-controlled, Randomized, doubleblINd, 12-week study with an open-label Extension (MARINE) trial]. Am J Cardiol. 2011;108:682-90.

14. Ballantyne CM, Bays HE, Kastelein JJ, Stein E, Isaacsohn JL, Braeckman RA, et al. Efficacy and safety of eicosapentaenoic acid ethyl ester (AMR101) therapy in statin-treated patients with persistent high triglycerides (from the ANCHOR study). Am J Cardiol. 2012;110:984-92.

15. Third Report of the National Cholesterol Education Program (NCEP). Expert Panel on Detection, Evaluation, and Treatment of High Blood Cholesterol in Adults (Adult Treatment Panel III) final report. Circulation. 2002;106:3143-421.

16. Jacobson TA, Ito MK, Maki KC, Orringer CE, Bays $\mathrm{HE}$, Jones $\mathrm{PH}$, et al. National Lipid Association recommendations for patient-centered management of dyslipidemia: part 1-executive summary. J Clin Lipidol. 2014;8:473-88.

17. Hilleman DE, Malesker MA. Potential benefits of icosapent ethyl on the lipid profile: case studies. Clin Med Insights Cardiol. 2014;8:13-5.

18. Castaldo RS. A retrospective case series of the lipid effects of switching from omega-3 fatty acid ethyl esters to icosapent ethyl in hyperlipidemic patients. Postgrad Med. 2014;126:268-73.

19. Berglund L, Brunzell JD, Goldberg AC, Goldberg IJ, Sacks F, Murad MH, et al. Evaluation and treatment of hypertriglyceridemia: an Endocrine Society clinical practice guideline. J Clin Endocrinol Metab. 2012;97:2969-89.

20. Friedewald WT, Levy RI, Fredrickson DS. Estimation of the concentration of low-density lipoprotein cholesterol in plasma, without use of the preparative ultracentrifuge. Clin Chem. 1972;18:499-502.

21. Brinton EA, Ballantyne CM, Bays HE, Kastelein JJ, Braeckman RA, Soni PN. Effects of icosapent ethyl on lipid and inflammatory parameters in patients with diabetes mellitus-2, residual elevated triglycerides (200-500 mg/dL), and on statin therapy at LDL-C goal: the ANCHOR study. Cardiovasc Diabetol. 2013;12:100.

22. Balk EM, Lichtenstein AH, Chung M, Kupelnick B, Chew P, Lau J. Effects of omega-3 fatty acids on 
serum markers of cardiovascular disease risk: a systematic review. Atherosclerosis. 2006;189:19-30.

23. GISSI Prevenzione Investigators. Dietary supplementation with n-3 polyunsaturated fatty acids and vitamin $\mathrm{E}$ after myocardial infarction: results of the GISSI-Prevenzione trial. Gruppo Italiano per lo Studio della Sopravvivenza nell'Infarto miocardico. Lancet. 1999;354:447-55.

24. GISSI-HF Investigators. Effect of n-3 polyunsaturated fatty acids in patients with chronic heart failure (the GISSI-HF trial): a randomised, double-blind, placebo-controlled trial. Lancet. 2008;372:1223-30.

25. Kromhout D, Giltay EJ, Geleijnse JM. n-3 fatty acids and cardiovascular events after myocardial infarction. N Engl J Med. 2010;363:2015-26.

26. ORIGIN Trial Investigators. n-3 fatty acids and cardiovascular outcomes in patients with dysglycemia. N Engl J Med. 2012;367:309-18.

27. Rauch B, Schiele R, Schneider S, Diller F, Victor N, Gohlke $\mathrm{H}$, et al. OMEGA, a randomized, placebocontrolled trial to test the effect of highly purified omega-3 fatty acids on top of modern guidelineadjusted therapy after myocardial infarction. Circulation. 2010;122:2152-9.

28. Yokoyama M, Origasa $H$, Matsuzaki M, Matsuzawa Y, Saito Y, Ishikawa Y, et al. Effects of eicosapentaenoic acid on major coronary events in hypercholesterolaemic patients (JELIS): a randomised open-label, blinded endpoint analysis. Lancet. 2007;369:1090-8.

29. Saito Y, Yokoyama M, Origasa H, Matsuzaki M, Matsuzawa Y, Ishikawa Y, et al. Effects of EPA on coronary artery disease in hypercholesterolemic patients with multiple risk factors: sub-analysis of primary prevention cases from the Japan EPA Lipid Intervention Study (JELIS). Atherosclerosis. 2008;200:135-40.

30. Mozaffarian D, Marchioli R, Macchia A, Silletta MG, Ferrazzi P, Gardner TJ, et al. Fish oil and postoperative atrial fibrillation: the Omega-3 Fatty Acids for Prevention of Post-operative Atrial Fibrillation (OPERA) randomized trial. JAMA. 2012;308:2001-11.

31. Larsson SC, Orsini N, Wolk A. Long-chain omega-3 polyunsaturated fatty acids and risk of stroke: a meta-analysis. Eur J Epidemiol. 2012;27:895-901.

32. The Risk and Prevention Study Collaborative Group. n-3 fatty acids in patients with multiple cardiovascular risk factors. $N$ Engl J Med. 2013;368:1800-8.

33. Matsuzaki M, Yokoyama M, Saito $Y$, Origasa $H$, Ishikawa Y, Oikawa $S$, et al. Incremental effects of eicosapentaenoic acid on cardiovascular events in statin-treated patients with coronary artery disease. Circ J. 2009;73:1283-90.

34. TG and HDL Working Group of the Exome Sequencing Project, National Heart, Lung, and Blood Institute. Loss-of-function mutations in APOC3, triglycerides, and coronary disease. N Engl J Med. 2014;371:22-31.

35. Jorgensen AB, Frikke-Schmidt R, Nordestgaard BG, Tybjaerg-Hansen A. Loss-of-function mutations in APOC3 and risk of ischemic vascular disease. N Engl J Med. 2014;371:32-41.

36. A study of AMR101 to evaluate its ability to reduce cardiovascular events in high risk patients with hypertriglyceridemia and on statin (REDUCE-IT). http://clinicaltrials.gov/show/NCT01492361. Accessed Nov 26, 2014. 